\title{
$\mathrm{n}$ 型 $\mathrm{SnS}$ 热电材料的制备与性能研究
}

\author{
黄志成 ${ }^{1,2}$, 姚 瑶 ${ }^{1}$, 裴 俊 $^{1,2}$, 董金峰 ${ }^{2}$, 张波萍 ${ }^{1}$, 李敬锋 ${ }^{2}$, 尚鹏鹏 ${ }^{3}$
}

(1. 北京科技大学 材料科学与工程学院, 北京新能源材料与技术重点实验室, 北京 100083 ; 2. 清华大学 材料学 院, 新型陶瓷与精细工艺国家重点实验室, 北京 100084; 3. 山东农业大学 化学与材料科学学院, 泰安 271018)

摘 要: SnS 由低毒、廉价、高丰度的元素组成, 在热电研究领域受到广泛关注。采用机械合金化(MA)结合放电等 离子烧结(SPS)工艺制备了 $\mathrm{n}$ 型 $\mathrm{SnS}_{1-x} \mathrm{Cl}_{x}(x=0,0.02,0.03,0.04,0.05,0.06)$ 多晶块体热电样品, 并研究了 $\mathrm{Cl}^{-}$掺杂量对 $\mathrm{SnS}$ 物相、微观结构以及电热输运性能的影响。结果表明: $\mathrm{Cl}^{-}$的引入会提高电子浓度, 使 $\mathrm{SnS}$ 由本征 $\mathrm{p}$ 型转变为 $\mathrm{n}$ 型半导体。随着 $\mathrm{Cl}^{-}$掺杂量的增加, $\mathrm{n}$ 型 $\mathrm{SnS}$ 半导体室温下的霍尔载流子浓度从 $6.31 \times 10^{14} \mathrm{~cm}^{-3}(x=0.03)$ 增加到 $7.27 \times$ $10^{15} \mathrm{~cm}^{-3}(x=0.06) \circ x=0.05$ 样品在 $823 \mathrm{~K}$ 取得最大的电导率为 $408 \mathrm{~S} \cdot \mathrm{m}^{-1}$, 同时具有较高的泽贝克系数为 $-553 \mu \mathrm{V} \cdot \mathrm{K}^{-1}$, 使其获得最大功率因子为 $1.2 \mu \mathrm{W} \cdot \mathrm{cm}^{-1} \cdot \mathrm{K}^{-2}$ 。Cl- 的掺入会引入点缺陷, 散射声子, 使晶格热导率 $\kappa_{\mathrm{lat}}$ 由 $0.67 \mathrm{~W} \cdot \mathrm{m}^{-1} \cdot \mathrm{K}^{-1}(x=0)$ 降至 $0.5 \mathrm{~W} \cdot \mathrm{m}^{-1} \cdot \mathrm{K}^{-1}(x=0.02)$ 。 $x=0.04$ 样品在 $823 \mathrm{~K}$ 获得了最大 $Z T$ 为 0.17 , 相比于 $x=0$ 样品 $(Z T \sim 0.1)$ 提高了 $70 \%$ 。

关 键 词: $\mathrm{SnS} ; \mathrm{Cl}$ 掺杂; $\mathrm{n}$ 型半导体; 热电性能

中图分类号: TB34 文献标识码: A

\section{Preparation and Thermoelectric Property of n-type SnS}

\author{
HUANG Zhi-Cheng ${ }^{1,2}$, YAO Yao ${ }^{1}$, PEI Jun ${ }^{1,2}$, DONG Jin-Feng ${ }^{2}$, \\ ZHANG Bo-Ping ${ }^{1}$, LI Jing-Feng ${ }^{2}$, SHANG Peng-Peng ${ }^{3}$
}

(1. The Beijing Municipal Key Laboratory of New Energy Materials and Technologies, School of Materials Science and Engineering, University of Science and Technology Beijing, Beijing 100083, China; 2. State Key Laboratory of New Ceramics and Fine Processing, School of Materials Science and Engineering, Tsinghua University, Beijing 100084, China; 3. College of Chemistry and Material Science, Shandong Agricultural University, Tai'an 271018, China)

\begin{abstract}
SnS composed of low toxicity, low-cost and earth-abundant elements, has extensive attention in the field of thermoelectric research. The n-type $\mathrm{SnS}_{1-x} \mathrm{Cl}_{x}(x=0,0.02,0.03,0.04,0.05,0.06)$ polycrystalline bulk thermoelectric samples were prepared by mechanical alloying (MA) combined with Spark Plasma Sintering (SPS). Effect of $\mathrm{Cl}^{-}$ amounts on the phase structure, microstructure and thermoelectric transport properties were systematically studied. Results show that introduction of $\mathrm{Cl}^{-}$enhances electron concentration which makes intrinsic p-type SnS change to n-type. With the amount of $\mathrm{Cl}^{-}$increasing, the Hall carrier concentration of n-type $\mathrm{SnS}$ semiconductor increases from $6.31 \times 10^{14} \mathrm{~cm}^{-3}(x=0.03)$ to $7.27 \times 10^{15} \mathrm{~cm}^{-3}(x=0.06)$ at room temperature. The maximum electrical conductivity of $408 \mathrm{~S} \cdot \mathrm{m}^{-1}$ and the relatively high Seebeck coefficient of $-553 \mu \mathrm{V} \cdot \mathrm{K}^{-1}$ are obtained at $823 \mathrm{~K}$ for $x=0.05$ sample, which produces the maximum power factor of $1.2 \mu \mathrm{W} \cdot \mathrm{cm}^{-1} \cdot \mathrm{K}^{-2}$. Addition of $\mathrm{Cl}^{-}$can introduce point defects to scatter phonons, which makes the lattice thermal conductivity reduce from $0.67 \mathrm{~W} \cdot \mathrm{m}^{-1} \cdot \mathrm{K}^{-1}(x=0)$ to $0.5 \mathrm{~W} \cdot \mathrm{m}^{-1} \cdot \mathrm{K}^{-1}(x=0.02)$. The highest $Z T \sim 0.17$ is obtained at $823 \mathrm{~K}$ for $x=0.04$ sample, which is $70 \%$ higher than that $(Z T \sim 0.1)$ of the pristine $\mathrm{SnS}$.
\end{abstract}

收稿日期：2018-06-29; 收到修改稿日期：2018-08-03

基金项目: 国家重点研发计划(2018YFB0703600); 国家自然科学基金(11474176)

National Key Research and Development Program of China (2018YFB0703600); National Natural Science Foundation of China (11474176).

作者简介: 黄志成(1994-), 男, 硕士研究生. E-mail: z.c.huang@foxmail.com

通讯作者: 张波萍, 教授. E-mail: bpzhang@ustb.edu.cn; 李敬锋, 教授. E-mail: jingfeng@mail.tsinghua.edu.cn 
Key words: SnS; Cl-doped; n-type semiconductor; thermoelectric properties

热电材料可以直接将热能和电能相互转换, 并 将此用于发电和制冷 ${ }^{[1]}$ 。热电材料的效率可以由无 量纲的热电优值 $(Z T)$ 给出: $Z T=\alpha^{2} \sigma T / \kappa$, 其中 $\alpha$ 是泽 贝克系数, $\sigma$ 是电导率, $T$ 是绝对温度, $\kappa$ 是热导率。 因此, 理想的热电材料要求有高功率因子 $\left(P F=\alpha^{2} \sigma\right)$ 和低热导率 $\kappa^{[2]}$ 。

IV-VI族半导体 SnS 由低毒、廉价、高丰度的元 素组成, 在热电研究领域受到广泛关注。 TAN 等 ${ }^{[3]}$ 报道 $\mathrm{SnS}-0.5 \% \mathrm{Ag}$ 多晶样品在 $923 \mathrm{~K}$ 取得 $Z T \sim 0.6$; ZHOU 等 ${ }^{[4]}$ 报道 $\mathrm{Na}$ 掺杂 $\mathrm{SnS}$ 多晶样品在 $873 \mathrm{~K}$ 取得 $Z T \sim 0.65$; WU 等 ${ }^{[5]}$ 报道单晶 $\mathrm{Na}_{0.02} \mathrm{Sn}_{0.98} \mathrm{~S}$ 在 $870 \mathrm{~K}$ 取得 $Z T \sim 1.1$; $\mathrm{HE}$ 等 ${ }^{[6]}$ 报道空穴掺杂单晶 $\mathrm{SnS}$ 在 $873 \mathrm{~K}$ 取 得最大 $Z T>1.0, Z T_{\mathrm{dev}}>0.57$, 且计算转化效率为 $10.4 \%$ 。以上均为 $\mathrm{p}$ 型 $\mathrm{SnS}$ 的相关研究 ${ }^{[3-6]}$, 而 $\mathrm{n}$ 型 $\mathrm{SnS}$ 热电性能鲜有报道。

为了制备 $\mathrm{n}$ 型 $\mathrm{SnS}$, 考虑到 $\mathrm{Cl}^{-}(0.181 \mathrm{~nm})$ 和 $\mathrm{S}^{2-}(0.184 \mathrm{~nm})$ 具有相似的离子半径, 本研究选择 $\mathrm{Cl}$ 作为掺杂剂。与 $\mathrm{SnS}$ 具有相同层状结构的 $\mathrm{SnSe}^{[7]}$, 通过掺杂 $\mathrm{BiCl}_{3}, \mathrm{Br}$, I 成功制备了 $\mathrm{n}$ 型 $\mathrm{SnSe}$ 材料 ${ }^{[8-10]}$ 。 本研究采用机械合金化(MA)结合放电等离子烧结 (SPS)成功制备了 $\mathrm{n}$ 型 $\mathrm{SnS}_{1-x} \mathrm{Cl}_{x}$, 并分析了材料的热 电性能。

\section{1 实验方法}

原料粉末 $\mathrm{Sn}(99.9 \%), \mathrm{S}(99.9 \%), \mathrm{SnCl}_{2}(99 \%)$ 按照 化学计量比 $\mathrm{SnS}_{1-x} \mathrm{Cl}_{x}(x=0,0.02,0.03,0.04,0.05$, 0.06)在手套箱中配料。使用行星式高能球磨机(中国, 南京南大仪器, QM-1SP2), 按球料比为 $20: 1$ 将不 锈钢球和原料装入不锈钢球磨罐中, 并充满保护气 (95\% Ar- $5 \% \mathrm{H}_{2}$ 混合气), 在 $450 \mathrm{r} / \mathrm{min}$ 转速下球磨 $15 \mathrm{~h}$, 合成 $\mathrm{SnS}_{1-x} \mathrm{Cl}_{x}$ 粉体。球磨后的粉体从手套箱中取出 并装入 $\phi 12 \mathrm{~mm}$ 石墨模具中, 采用放电等离子烧结 (SPS)。烧结温度为 $853 \mathrm{~K}$, 烧结压力为 $50 \mathrm{MPa}$, 保 温保压时间为 $5 \mathrm{~min}$ 。最终得到直径为 $12 \mathrm{~mm}$, 高度 为 $10 \mathrm{~mm}$ 的圆柱形块体样品。

采用 X 射线衍射仪(德国, Bruker, D8 Advance) 表征 $\mathrm{SnS}_{1-x} \mathrm{Cl}_{x}$ 块体相结构, 以 $\mathrm{Cu}$ 靶为光源。采用 场发射电子显微镜(德国, Zeiss Merlin, FESEM)观察 样品断口的微观组织形貌。使用泽贝克系数与电阻 率综合测量系统(日本, Ulvac-Riko, ZEM-2)测试泽 贝克系数 $(\alpha)$ 和电阻率 $(\rho)$ 。热导率 $\kappa$ 通过公式 $\kappa=$ $D C_{\mathrm{p}} d$ 计算得到, 其中 $D$ 为热扩散系数, $C_{\mathrm{p}}$ 为热容, $d$
为样品密度。热扩散系数 $D$ 通过激光闪射法(德国, Netzsch, LFA457)测量, $C_{\mathrm{p}}$ 采用文献值 ${ }^{[11]}, d$ 采用阿 基米德法测量。载流子浓度 $n=1 /\left(e R_{\mathrm{H}}\right)$, 迁移率 $\mu=$ $\sigma R_{\mathrm{H}}$, 其中 $e$ 为电子电荷 $\left(e=1.6 \times 10^{-19} \mathrm{C}\right)$, 霍尔系数 $R_{\mathrm{H}}$ 用霍尔测试系统(日本, Toyo, ResiTest 8400)测量。 样品的热电性能测试方向均平行于 SPS 压力方向。

\section{2 结果与讨论}

\section{1 物相组成及微观结构}

图 1(a)给出了 $\mathrm{SnS}_{1-x} \mathrm{Cl}_{x}(x=0,0.02,0.03,0.04$, $0.05,0.06$ )块体平行于压力方向样品的 XRD 图谱。 当 $x=0$ 时, XRD 中所有衍射峰均与 $\mathrm{SnS}$ 标准卡片 (PDF\#39-0354)吻合, 在 XRD 检测极限内未检测到 杂质相，表明利用 MA+SPS 成功制备了 $\mathrm{SnS}$ 纯相块 体; 当 $0.02 \leqslant x \leqslant 0.04$ 时, 样品仍为 $\mathrm{SnS}$ 单相; 当 $0.05 \leqslant x \leqslant 0.06$ 时, 出现杂质相, 对应单质 $\mathrm{Sn}$ 特征峰 $(\diamond, \mathrm{PDF} \# 86-2264)$, 表明析出了单质 $\mathrm{Sn}$ 。为了讨论 $\mathrm{Cl}$ 掺杂量 $(x$ 值)的变化对主相 $\mathrm{SnS}$ 晶格的影响, 将 $\mathrm{SnS}$ 相(111)和(042)衍射峰放大, 如图 1(b) (c) 所示。 (111)和(042)衍射峰均具有相同的趋势。当 $0 \leqslant x \leqslant 0.04$ 时, 衍射峰向大角度偏移, 表明晶格常数减小; 当 $0.04<x \leqslant 0.06$ 时, 衍射峰向标准衍射峰位回归, 表 明晶格常数回复。

图 2 给出了晶格常数和晶胞体积随 $x$ 值的变化 曲线。当 $0 \leqslant x \leqslant 0.04$ 时, 晶格常数和晶胞体积呈减 小趋势; 当 $0.04 \leqslant x \leqslant 0.06$ 时, 晶格常数和晶胞体积 呈增大趋势。

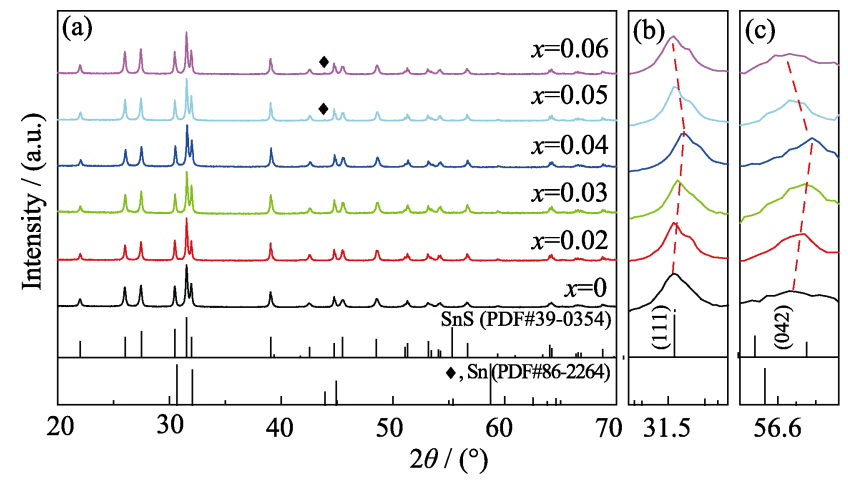

图 $1 \mathrm{SnS}_{1-x} \mathrm{Cl}_{x}(x=0,0.02,0.03,0.04,0.05,0.06)$ 块体平行于 压力方向样品的 XRD 图谱

Fig. 1 XRD patterns of the $\mathrm{SnS}_{1-x} \mathrm{Cl}_{x}(x=0,0.02,0.03,0.04$, $0.05,0.06$ ) bulks at (a) $20^{\circ}-70^{\circ}$, (b) $31.3^{\circ}-31.8^{\circ}$ and (c) $56.5^{\circ}-$ $56.8^{\circ}$, which was measured along the hot pressing direction 


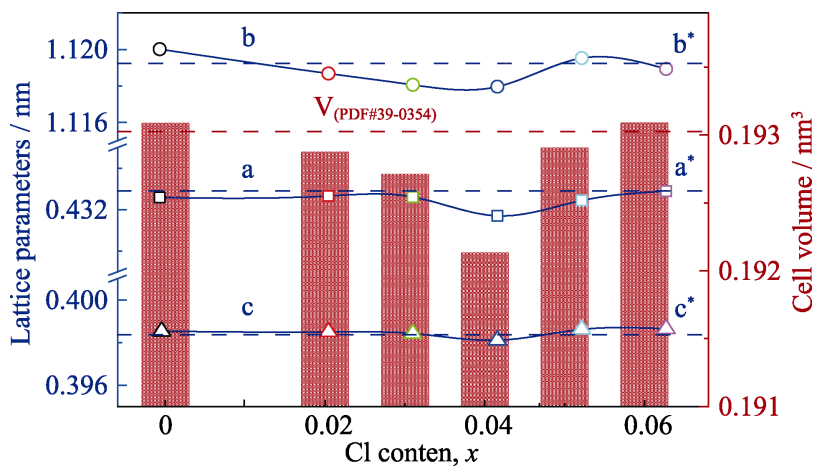

图 $2 \mathrm{SnS}_{1-x} \mathrm{Cl}_{x}(x=0,0.02,0.03,0.04,0.05,0.06)$ 块体的晶格 常数(点), 晶胞体积(柱状图)及其标准卡片(PDF\#39-0354)值 (虚线)随 $x$ 值变化图

Fig. 2 Lattice parameters (dots) and cell volume (histogram) of the $\mathrm{SnS}_{1-x} \mathrm{Cl}_{x}(x=0,0.02,0.03,0.04,0.05,0.06)$ bulks samples along with those of the standard card (dashed lines) for SnS (PDF\#39-0354)

在制备 $\mathrm{SnS}$ 过程中，可能会存在 $\mathrm{Sn} 、 \mathrm{~S}$ 的挥发 和损失，其缺陷方程如下：

$$
\begin{gathered}
\mathrm{SnS} \rightarrow \mathrm{Sn}_{1-x} \mathrm{~S}+x \mathrm{Sn} \uparrow \text { or loss }+x \mathrm{~V}_{\mathrm{Sn}}^{\prime \prime}+2 x \mathrm{~h}^{\circ} \\
\mathrm{SnS} \rightarrow \mathrm{SnS}_{1-x}+x \mathrm{~S} \uparrow \text { or loss }+x \mathrm{~V}^{*{ }_{\mathrm{S}}}+2 x \mathrm{e}^{\prime}
\end{gathered}
$$

方程(1)表明 $\mathrm{Sn}$ 的挥发或损失产生 $x$ 个 $\mathrm{Sn}$ 空位 $\left(V^{\prime \prime}{ }_{\mathrm{Sn}}\right)$ 和 $2 x$ 个空穴 $\left(\mathrm{h}^{\circ}\right)$ ，会增加空穴载流子浓度; 方 程(2)表明 $\mathrm{S}$ 挥发或损失产生 $x$ 个 $\mathrm{S}$ 空位 $\left(V^{*} \mathrm{~S}\right)$ 和 $2 x$ 个电子 $\left(\mathrm{e}^{\prime}\right)$ 。由于 $\mathrm{Sn} 、 \mathrm{~S}$ 挥发产生的 $V^{\prime \prime}{ }_{\mathrm{Sn}}$ 和 $V^{\circ{ }_{\mathrm{S}}}$ 会减 小晶胞体积。

当 $\mathrm{Cl}^{-}$进入 $\mathrm{SnS}$ 中时, 可能存在进入 $\mathrm{S}$ 置换位和 间隙位两种情况，其具体的缺陷方程如下:

$$
\begin{aligned}
& \mathrm{SnCl}_{2} \stackrel{\mathrm{SnS}}{\longrightarrow} 2 \mathrm{Cl}_{\mathrm{S}}^{\circ}+\mathrm{Sn}_{\mathrm{Sn}}+2 \mathrm{e}^{\prime} \\
& \mathrm{SnCl}_{2} \stackrel{\mathrm{SnS}}{\longrightarrow} 2 \mathrm{Cl}_{i}^{\prime}+\mathrm{Sn}_{\mathrm{Sn}}+2 \mathrm{~h}^{\circ}
\end{aligned}
$$

方程(3)表明 1 个 $\mathrm{Cl}^{-}$置换 $\mathrm{S}^{2-}$ 提供 1 个电子 $\left(e^{\prime}\right)$, 从而提高电子载流子浓度, 同时由于 $\mathrm{Cl}^{-}$的离子半 径 $(0.181 \mathrm{~nm})$ 略小于 $\mathrm{S}^{2-}(0.184 \mathrm{~nm}), \mathrm{Cl}^{-}$进入 $\mathrm{S}^{2-}$ 位会 减小晶格常数和晶胞体积; 方程(4)表明 $\mathrm{Cl}^{-}$进入间 隙位置会产生空穴 $\left(h^{\circ}\right)$, 增大空穴载流子浓度, 同时 会导致晶格增大。当 $0 \leqslant x \leqslant 0.04$ 时, 晶格常数先减
小，表明 $\mathrm{Cl}^{-}$可能优先进入 $\mathrm{S}^{2-}$ 位。

进一步增大 $\mathrm{Cl}$ 掺杂量时, 析出少量的 $\mathrm{Sn}$ 单质, 这可能是由于: $\mathrm{Cl} 、 \mathrm{~S} 、 \mathrm{Sn}$ 的电负性关系为 $\mathrm{Cl}(3.16)>$ $\mathrm{S}(2.58)>\mathrm{Sn}(1.96), \mathrm{Cl}$ 与 $\mathrm{Sn}$ 的电负性差值更大, 加入 $\mathrm{Cl}$ 会进一步促进 $\mathrm{S}$ 的挥发, 进而导致 Sn 过饱和析 出。Sn 的过饱和析出, 会填补 $V^{\prime \prime}{ }_{\mathrm{Sn}}$, 降低空穴浓度, 提高电子载流子浓度，同时增大晶胞体积。

表 1 给出了 $\mathrm{SnS}_{1-x} \mathrm{Cl}_{x}$ 的名义成分、实际成分及 室温下的霍尔性质。当 $x=0$ 时, $\mathrm{SnS}$ 的实际成分为 $\mathrm{SnS}_{0.97}$, 表明 $V^{*}{ }_{\mathrm{S}}$ 在 $\mathrm{SnS}$ 占主导。根据缺陷方程(2), $V^{\circ}{ }_{\mathrm{S}}$ 的产生会增加电子载流子浓度, $\mathrm{SnS}$ 应该表现出 $\mathrm{n}$ 型半导体的性质。但此时 $R_{\mathrm{H}}$ 为正值，表现出 $\mathrm{p}$ 型 半导体的性质。这说明在 $\mathrm{SnS}$ 制备过程中, 还伴随 着 $\mathrm{Sn}$ 空位的形成。 $V^{\prime \prime}{ }_{\mathrm{Sn}}$ 与 $V^{*}{ }_{\mathrm{S}}$ 的共同作用导致 $x=0$ 的样品表现出 $\mathrm{p}$ 型半导体的性质。

随着 $\mathrm{Cl}$ 掺杂量 $(x)$ 增加, $R_{\mathrm{H}}$ 先增大后减小, 在 $x=0.02$ 附近处发生 $\mathrm{p}-\mathrm{n}$ 转变。当 $x \geqslant 0.03$ 时, 随着 $x$ 值的增大, 载流子浓度逐渐提高, 说明 $\mathrm{Cl}^{-}$优先进入 $\mathrm{S}^{2-}$ 位，这与 XRD 的分析结果相一致。

$(\mathrm{S}+\mathrm{Cl}) / \mathrm{Sn}$ 的值随着 $x$ 值增大而增大，说明 $V^{*}{ }_{\mathrm{S}}$ 不断增加, 导致 $\mathrm{Sn}$ 过饱和析出, 会填补 $V^{\prime \prime}{ }_{\mathrm{Sn}}$, 降低空 穴浓度, 增加电子载流子浓度, 同时增大晶胞体积。

综上所述：随着 $\mathrm{Cl}$ 掺杂量 $(x)$ 增大，可能同时存 在三种过程: (1) $\mathrm{Sn}$ 和 $\mathrm{S}$ 的挥发形成 $V^{\prime \prime}{ }_{\mathrm{Sn}}$ 和 $V^{\circ}{ }_{\mathrm{S}}$, 导 致晶格减小; (2) $\mathrm{Cl}^{-}$置换 $\mathrm{S}^{2-}$, 导致晶格减小; (3)单质 $\mathrm{Sn}$ 填补部分 $V^{\prime \prime}{ }_{\mathrm{Sn}}$, 导致晶格增大, 三者共同作用导 致衍射峰偏移、晶格常数及晶胞体积变化。当 $x=0$ 时, $\mathrm{S}$ 的挥发占主导, 形成了 $V^{\circ} \mathrm{s}$; 当 $0.02 \leqslant x \leqslant 0.04$ 时, $\mathrm{Cl}^{-}$置换 $\mathrm{S}^{2-}$ 占主导作用, 衍射峰向大角度偏移、 晶格常数和晶胞体积减小, 同时电子载流子浓度增 大; 当 $x>0.04$ 时，单质 $\mathrm{Sn}$ 填补部分 $V^{\prime} \mathrm{Sn}$ 占主导作用, 衍射峰回归、晶格常数和晶胞体积增大, 空穴载流 子浓度减小，电子载流子浓度增大。

图 3 给出了 $x=0.03$ 样品的(a)背散射(BSE)照片, (b)区域 1,2 的 EDS 图谱, 以及(c, d) S, Sn 元素分布 图。从图 3(a)中可以观察到有白色部分和灰色部分

表 $1 \mathrm{SnS}_{1-x} \mathrm{Cl}_{x}$ 名义成分、实际成分及室温下霍尔系数, 载流子浓度, 迁移率

Table 1 Room temperature nominal composition, real composition, Hall coefficient, Hall carrier concentration, and mobility for $\mathrm{SnS}_{1-x} \mathrm{Cl}_{x}$

\begin{tabular}{cccccc}
\hline$x$ & Nominal comp. & Real comp. & $R_{\mathrm{H}} /\left(\mathrm{cm}^{3} \cdot \mathrm{C}^{-1}\right)$ & $n_{\mathrm{H}} / \mathrm{cm}^{-3}$ & $\mu_{\mathrm{H}} /\left(\mathrm{cm}^{2} \cdot \mathrm{V}^{-1} \cdot \mathrm{s}^{-1}\right)$ \\
\hline 0 & $\mathrm{SnS}$ & $\mathrm{SnS}_{0.97}$ & $1.55 \times 10^{3}$ & $4.03 \times 10^{15}$ & 1.29 \\
0.02 & $\mathrm{SnS}_{0.98} \mathrm{Cl}_{0.02}$ & $\mathrm{SnS}_{0.95} \mathrm{Cl}$ & $1.43 \times 10^{5}$ & $4.35 \times 10^{13}$ & 2.83 \\
0.03 & $\mathrm{SnS}_{0.97} \mathrm{Cl}_{0.03}$ & $\mathrm{SnS}_{0.97} \mathrm{Cl}_{0.005}$ & $-9.9 \times 10^{3}$ & $6.31 \times 10^{14}$ & 2.34 \\
0.04 & $\mathrm{SnS}_{0.96} \mathrm{Cl}_{0.04}$ & $\mathrm{SnS}_{0.95} \mathrm{Cl}_{0.009}$ & $-7.19 \times 10^{3}$ & $8.68 \times 10^{14}$ & 3.04 \\
0.05 & $\mathrm{SnS}_{0.95} \mathrm{Cl}_{0.05}$ & $\mathrm{SnS}_{0.93} \mathrm{Cl}_{0.021}$ & $-9.22 \times 10^{2}$ & $6.78 \times 10^{15}$ & 3.18 \\
0.06 & $\mathrm{SnS}_{0.94} \mathrm{Cl}_{0.06}$ & $\mathrm{SnS}_{0.92} \mathrm{Cl}_{0.022}$ & $-8.6 \times 10^{2}$ & $7.27 \times 10^{15}$ & 3.60 \\
\hline
\end{tabular}




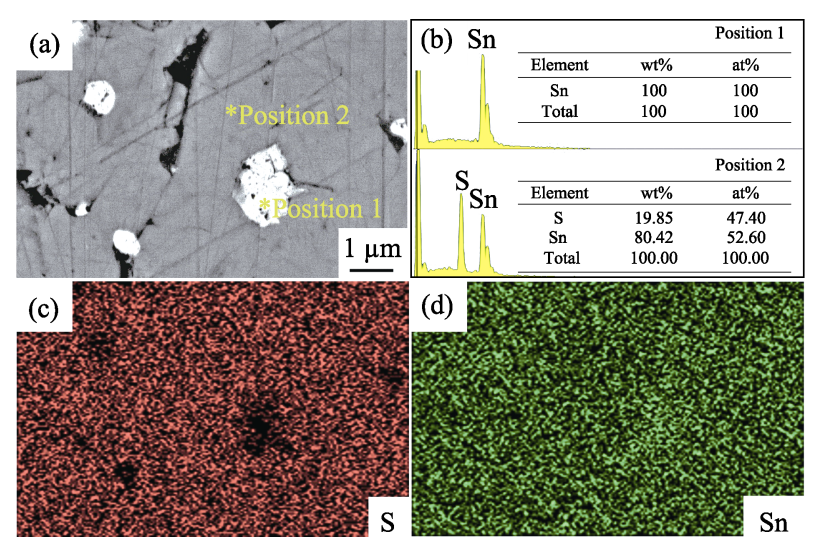

图 $3 x=0.03$ 样品的(a)背散射(BSE)照片, (b) 区域 1, 2 的 EDS 图谱, 以及(c) (d) S, Sn 元素分布图

Fig. 3 (a) The back-scattered electron (BSE) image of the polished surface, (b) EDS spectra taken from position 1 and 2 and (c) (d) elemental distributions of S, Sn for $x=0.03$ sample

两个部分(黑色部分为凹坑和抛光划痕)。由图 3(b) 两个部分的元素分析可知: 白色部分(Position 1)为 第二相 $\mathrm{Sn}$ 单质, 灰色部分(Position 2)为 $\mathrm{SnS}$ 基体 相。图 3(c) (d)的元素分布分析(EDS mapping)显示, 白色部分有 $\mathrm{Sn}$ 分布而没有 $\mathrm{S}$ 分布, 进一步证实白色 部分为析出的单质 $\mathrm{Sn}$ 。同时 EDS 元素分析(表 1)显 示: 当 $x \geqslant 0.03$ 时, 元素 $\mathrm{S}$ 实际成分较名义成分的减 少量随 $x$ 值的增大而增大, 可以推测出 $\mathrm{SnS}_{1-x} \mathrm{Cl}_{x}$ 块 体在 $x \geqslant 0.03$ 时开始逐渐析出单质 $\mathrm{Sn}$ 第二相, 并影 响块体电热性能。这里单质 $\mathrm{Sn}$ 从 $x=0.03$ 开始析出, 而 XRD 结果显示 $x \geqslant 0.05$ 时衍射峰向标准衍射峰位 回归。在前述三种过程共同作用下, 衍射峰表现取 决于哪种过程占主导。EDS 结果和 XRD 结果的外 在表现有所区别，但其内在反应过程一致。

图 4(a) (f)给出了 $\mathrm{SnS}_{1-x} \mathrm{Cl}_{x}$ 块体的断口形貌, 从图中可以看出, 所有样品都具有层片状组织, 未 观察到明显取向。相比于 $x=0$ 样品, $x \geqslant 0.02$ 样品晶 粒增大。图 $4(\mathrm{~g})$ 为 $\mathrm{SnS}_{1-x} \mathrm{Cl}_{x}$ 块体平均晶粒尺寸和相 对密度随 $x$ 值变化曲线, 平均晶粒尺寸随着 $x$ 值的 增大呈增大趋势: 由 $0.85 \mu \mathrm{m}(x=0)$ 增大到 $1.29 \mu \mathrm{m}$ $(x=0.06) 。 \mathrm{SnS}_{1-x} \mathrm{Cl}_{x}$ 晶粒尺寸随 $x$ 值的增大而增大, 可以分为第二相出现前 $(0 \leqslant x \leqslant 0.02)$ 和出现后 $(0.03 \leqslant$ $x \leqslant 0.06$ )两段进行分析, 当 $0 \leqslant x \leqslant 0.02$ 时, 掺杂原子 $\mathrm{Cl}$ 对晶格起活化作用, 增大晶粒尺寸; 当 $0.03 \leqslant x \leqslant$ 0.06 时, 晶粒尺寸增大趋势较 $0 \leqslant x \leqslant 0.02$ 明显减 缓。拟合直线(图 4(g) 黑实线)由斜率 $k=13(0 \leqslant x \leqslant$ $0.02)$ 降低为斜率 $k=3.5(0.03 \leqslant x \leqslant 0.06)$ 。这主要是由 于析出的单质 $\mathrm{Sn}$ 的钉扎作用抑制了晶粒尺寸增大。 掺杂原子 $\mathrm{Cl}$ 的活化作用和第二相 $\mathrm{Sn}$ 单质的钉扎作 用共同起作用, 使得 $x \geqslant 0.03$ 时晶粒尺寸缓慢增大。 此外, 所有样品相对密度都在 $90 \%$ 以上。
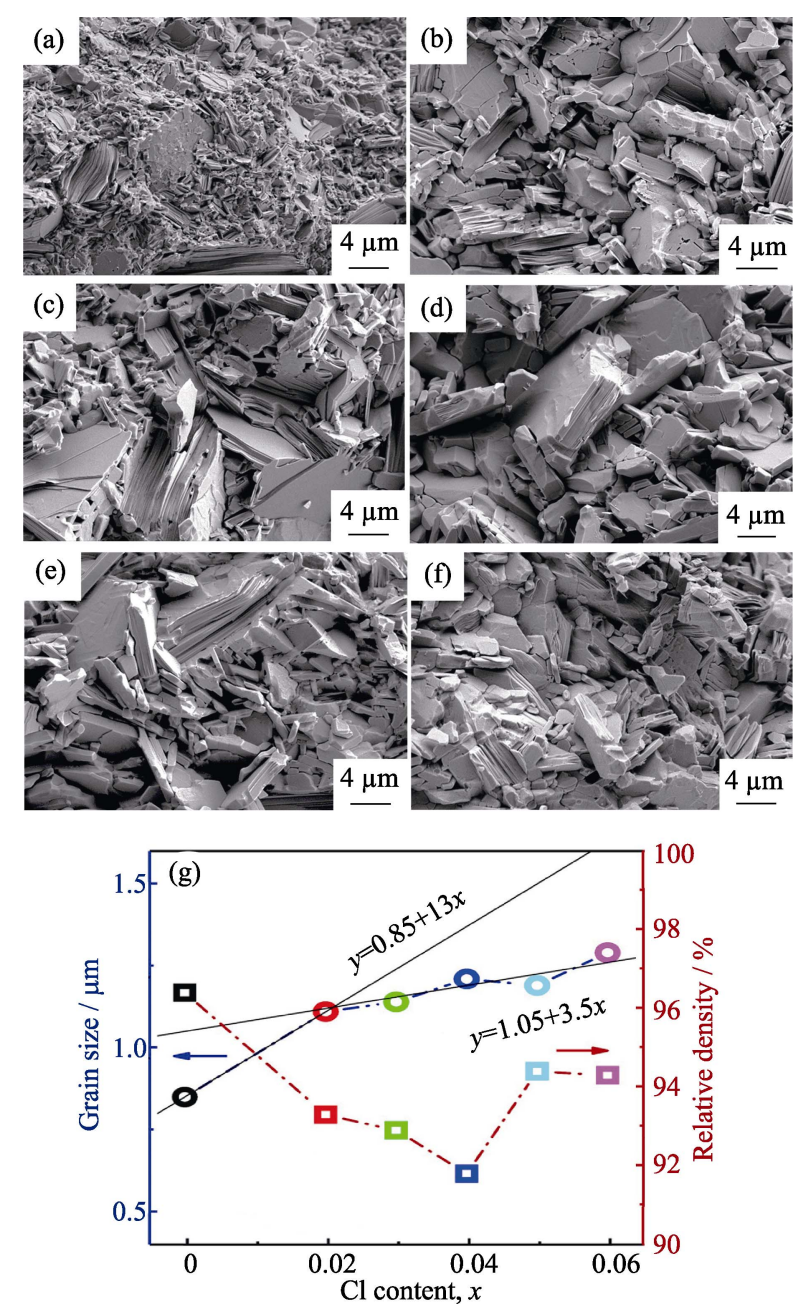

图 $4 \mathrm{SnS}_{1-x} \mathrm{Cl}_{x}$ 块体的断口 SEM 照片 (a) $x=0$, (b) $x=0.02$, (c) $x=0.03$, (d) $x=0.04$, (e) $x=0.05$, (f) $x=0.06$; (g) $\mathrm{SnS}_{1-x} \mathrm{Cl}_{x}$ 晶粒尺 寸(圆圈)和相对密度(方块)随 $x$ 值变化曲线

Fig. 4 SEM images of the fractured surface morphologies for (a) $x=0$, (b) $x=0.02$, (c) $x=0.03$, (d) $x=0.04$, (e) $x=0.05$, (f) $x=$ 0.06 , and $(\mathrm{g})$ change of grain size (circle) and relative density (square) with $x$ for $\mathrm{SnS}_{1-x} \mathrm{Cl}_{x}$ bulk samples

\section{2 电性能}

图 5(a)给出了 $\mathrm{SnS}_{1-x} \mathrm{Cl}_{x}$ 块体的泽贝克系数 $\alpha$ 随 温度变化曲线。当 $x=0$ 时, 样品为 $\mathrm{p}$ 型 $\mathrm{SnS}, \alpha$ 在测 试温度区间内呈先增大后减小趋势。 $x=0.02$ 样品在 423 473 K 测试温度范围出现 $\mathrm{p}-\mathrm{n}$ 转变, $\mathrm{Cl}^{-}$置换 $\mathrm{S}^{2-}$ 提供电子, 且升温后热激发出更多电子; $x \geqslant 0.03$ 样 品在整个测量温度区间内的泽贝克系数 $\alpha$ 为负值, 且 霍尔系数 $R_{\mathrm{H}}$ 为负值, 表明其为 $\mathrm{n}$ 型半导体, 且随着温 度升高, 泽贝克系数绝对值 $|\alpha|$ 呈减小趋势。

泽贝克系数 $\alpha(323 \mathrm{~K})$ 的行为可以通过泽贝克系 数 $\alpha$ 与载流子浓度 $n_{\mathrm{H}}$ 关系分析, 如图 5(b)所示。当 $0.03 \leqslant x \leqslant 0.04$ 时, 泽贝克系数绝对值 $|\alpha|$ 随着载流 子浓度 $n_{\mathrm{H}}$ 增加而增大, 可以用式 $\alpha=\frac{\alpha_{\mathrm{p}} \sigma_{\mathrm{p}}+\alpha_{\mathrm{n}} \sigma_{\mathrm{n}}}{\sigma_{\mathrm{p}}+\sigma_{\mathrm{n}}}{ }^{[12]}$ 

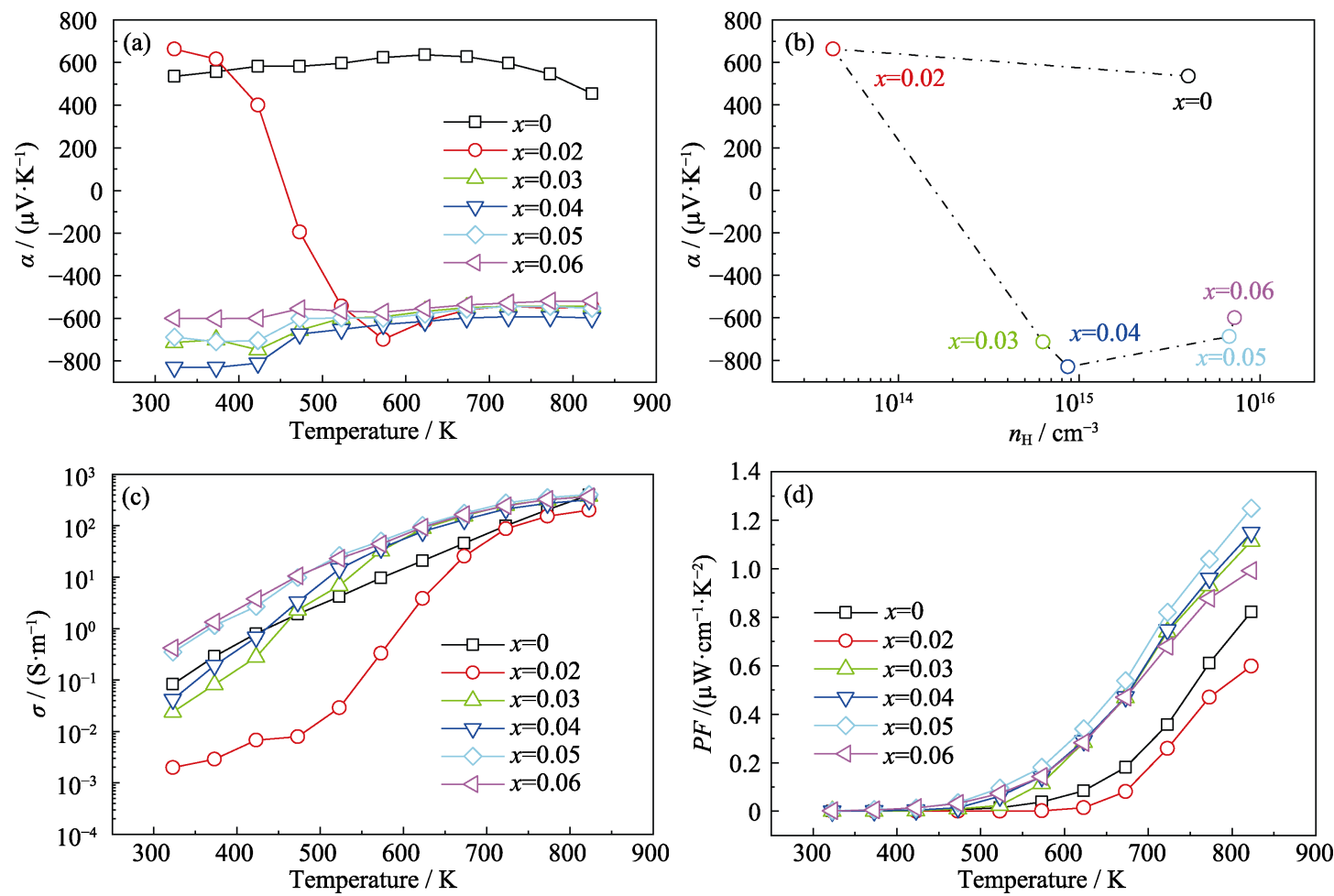

图 $5 \mathrm{SnS}_{1-x} \mathrm{Cl}_{x}(x=0,0.02,0.03,0.04,0.05,0.06)$ 块体的(a)泽贝克系数, (b)载流子浓度泽贝克系数关系曲线, (c)电导率和(d)功率因子随温度变化曲线

Fig. 5 Temperature dependence of (a) Seebeck coefficient, (b) relation curve of Hall carrier concentration - Seebeck coefficient, (c) electrical conductivity, and (d) power factor for $\mathrm{SnS}_{1-x} \mathrm{Cl}_{x}(x=0,0.02,0.03,0.04,0.05,0.06)$

来解释: 泽贝克系数 $\alpha$ 由 $\mathrm{p}$ 型空穴载流子贡献的正 泽贝克系数 $\alpha_{\mathrm{p}}$ 和 $\mathrm{n}$ 型电子载流子贡献的负泽贝克系 数 $\alpha_{\mathrm{n}}$ 构成, 当 $0.03 \leqslant x \leqslant 0.04$ 时, 此时少子空穴浓度 仍然较高, 随着 $x$ 值的增大 $\alpha_{\mathrm{p}}$ 的贡献减少, 表现为 泽贝克系数绝对值 $|\alpha|$ 增加(该现象在 I 掺杂 $\mathrm{SnSe}$ 中 也曾报道 ${ }^{[10]}$ ); 当 $0.05 \leqslant x \leqslant 0.06$ 时, 载流子浓度增 加，泽贝克系数绝对值 $|\alpha|$ 降低。

图 5(c)给出了 $\mathrm{SnS}_{1-x} \mathrm{Cl}_{x}$ 块体的电导率 $\sigma$ 随温度 变化图。所有样品电导率 $\sigma$ 随着温度升高而增大, 呈现半导体行为; 随着 $x$ 值的增大, $323 \mathrm{~K}$ 时电导率 $\sigma$ 呈现先下降后上升的趋势, 电导率 $\sigma(323 \mathrm{~K})$ 从 $0.083 \mathrm{~S} \cdot \mathrm{m}^{-1}(x=0)$ 下降到 $0.0019 \mathrm{~S} \cdot \mathrm{m}^{-1}(x=0.02)$ 再上 升到 $0.42 \mathrm{~S} \cdot \mathrm{m}^{-1}(x=0.06)$ 。电导率 $\sigma(\sigma=n e \mu)$ 的行为可 以通过表 1 中霍尔载流子浓度 $n_{\mathrm{H}}$ 和迁移率 $\mu$ 的行为 来解释: 由于掺入的施主杂质 $\mathrm{Cl}^{-}$提供了电子，电子 载流子起主导作用, 载流子浓度表现为先减小后增大, 二者中载流子浓度占主导作用导致电导率先下降后 上升。但样品载流子浓度仍然较低(从 $6 \times 10^{14} \mathrm{~cm}^{-3}$ $(x=0.03)$ 增大到 $\left.7 \times 10^{15} \mathrm{~cm}^{-3}(x=0.06)\right)$, 这是因为 $\mathrm{Cl}$ 在 MA/SPS 过程中损失造成 $\mathrm{Cl}$ 在样品中的实际含量 (见表 1 )较低。值得一提的是, 当 $x \geqslant 0.03$ 时, 载流 子浓度增加的同时迁移率也提高, 这可能是由于晶 粒尺寸增大 ${ }^{[13]}$ 造成的。
图 5(d)给出了 $\mathrm{SnS}_{1-x} \mathrm{Cl}_{x}$ 块体的功率因子随温度 变化图。功率因子 $P F=\alpha^{2} \sigma$, 所有样品功率因子 $P F$ 随着温度升高而增大。随着 $x$ 值的增大, 样品功率 因子 $P F(823 \mathrm{~K})$ 呈先下降后上升趋势。 $x=0.02$ 样品 功率因子 $P F$ 比 $x=0$ 样品低; $x \geqslant 0.03$ 样品的功率因 子 $P F$ 均比 $x=0$ 样品提高; $x=0.05$ 样品的功率因子 $P F$ 在 $823 \mathrm{~K}$ 取得最大值, 达到 $1.2 \mu \mathrm{W} \cdot \mathrm{cm}^{-1} \cdot \mathrm{K}^{-2}$ 。

\section{3 热性能}

图 6(a)给出了 $\mathrm{SnS}_{1-x} \mathrm{Cl}_{x}$ 块体的热扩散系数 $D$ 随 温度变化图, 所有样品的热扩散系数 $D$ 随温度升高 而降低。在测试温度区间内, $x \geqslant 0.02$ 样品的热扩散 系数 $D$ 均比 $x=0$ 样品低; $x \geqslant 0.02$ 样品的热扩散系数 $D$ 随着 $x$ 值的增大呈现上升趋势。

图 6(b)给出了 $\mathrm{SnS}_{1-x} \mathrm{Cl}_{x}$ 总热导率 $\kappa_{\mathrm{tot}}$ 随温度变 化图。总热导率 $\kappa_{\mathrm{tot}}\left(\kappa_{\mathrm{tot}}=D C_{\mathrm{p}} d\right)$ 变化趋势与热扩散系 数 $D$ 变化趋势类似。 $x \geqslant 0.02$ 样品的总热导率 $\kappa_{\mathrm{tot}}$ 均比 $x=0$ 样品低, 且随 $x$ 值的增大呈现上升趋势。

在 $823 \mathrm{~K}$ 时, 总热导率 $\kappa_{\mathrm{tot}}$ 由 $0.68 \mathrm{~W} \cdot \mathrm{m}^{-1} \cdot \mathrm{K}^{-1}(x=0)$ 降至 $0.56 \mathrm{~W} \cdot \mathrm{m}^{-1} \cdot \mathrm{K}^{-1}(x=0.02)$ 。总热导率 $\kappa_{\mathrm{tot}}$ 由电子 热导率 $\kappa_{\mathrm{e}}$ 和晶格热导率 $\kappa_{\text {lat }}$ 组成, 为了进一步解释 材料的热输运行为, 下面对电子热导率 $\kappa_{\mathrm{e}}$ 和晶格热 导率 $\kappa_{\text {lat }}$ 进行讨论。

由于电导率 $\sigma$ 较低导致电子热导率 $\left(\kappa_{\mathrm{e}}=L \sigma T\right)$ 较 

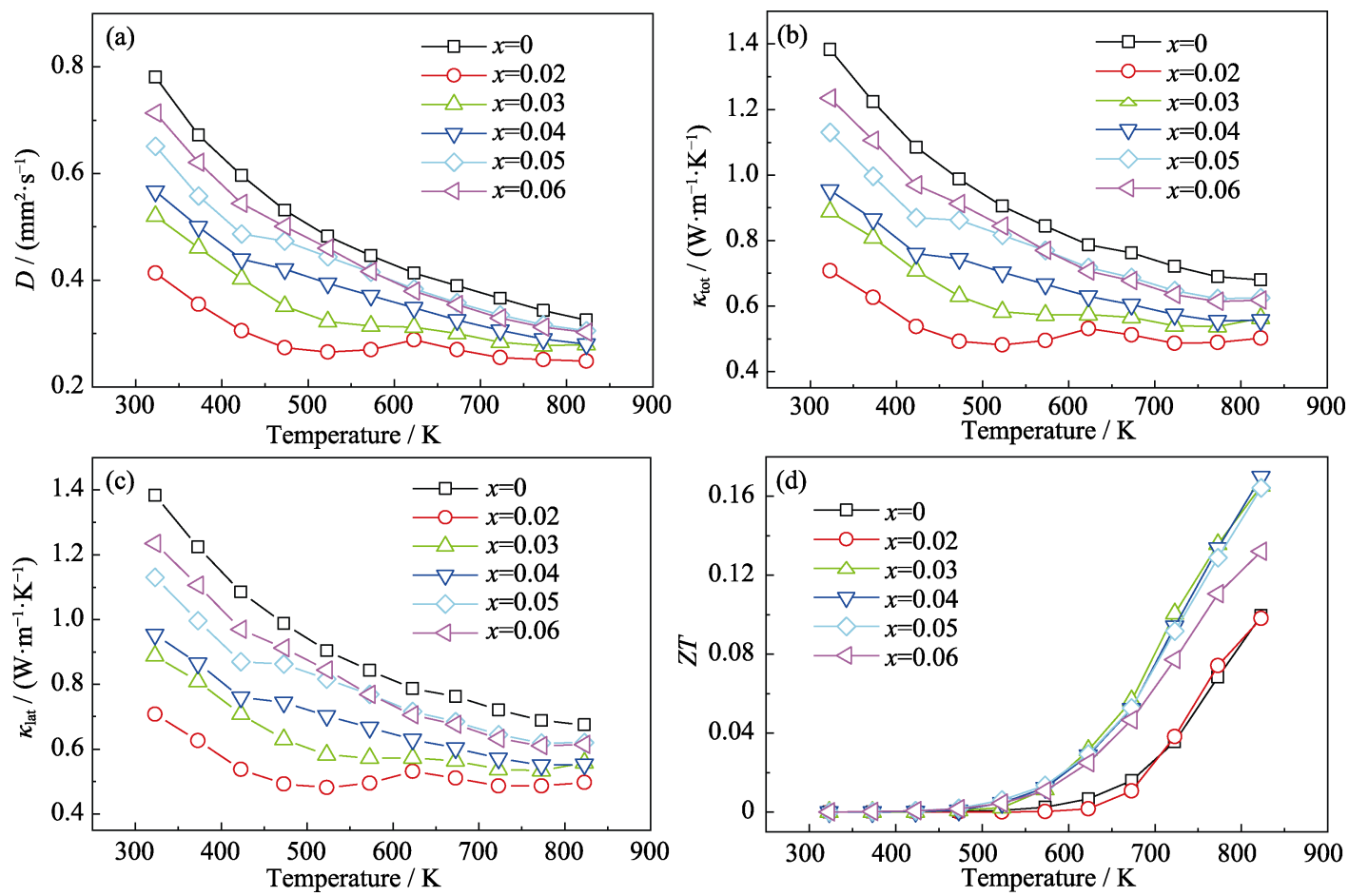

图 $6 \mathrm{SnS}_{1-x} \mathrm{Cl}_{x}(x=0,0.02,0.03,0.04,0.05,0.06)$ 块体(a)热扩散系数,

(b)总热导率, (c)晶格热导率和 (d)热电优值随温度变化曲线

Fig. 6 Temperature dependence of (a) thermal diffusivity, (b) total thermal conductivity, (c) lattice thermal conductivity, and (d) $Z T$ for $\mathrm{SnS}_{1-x} \mathrm{Cl}_{x}(x=0,0.02,0.03,0.04,0.050 .06$ )

低 $\left(823 \mathrm{~K}\right.$ 时约为 $\left.5 \times 10^{-3} \mathrm{~W} \cdot \mathrm{m}^{-1} \cdot \mathrm{K}^{-1}\right)$, 晶格热导率 $\left(\kappa_{\mathrm{lat}}=\kappa_{\mathrm{tot}}-\kappa_{\mathrm{e}}\right)$ 在总热导率 $\kappa_{\mathrm{tot}}$ 占主导地位。图 6(c)给出 了 $\mathrm{SnS}_{1-x} \mathrm{Cl}_{x}$ 晶格热导率 $\kappa_{\mathrm{lat}}$ 随温度变化图。相比于 $x=0$ 样品, $x=0.02$ 样品的晶格热导率 $\kappa_{\text {lat }}$ 下降, 可能 是掺入 $\mathrm{Cl}^{-}$引入了点缺陷散射声子导致的; 随着 $x$ 值 的进一步增大, 晶格热导率 $\kappa_{\mathrm{lat}}$ 呈上升趋势。这可能 是由晶粒尺寸逐渐增加, 导致晶界散射减少, 同时 高热导率 $\mathrm{Sn}\left(68 \mathrm{~W} \cdot \mathrm{m}^{-1} \cdot \mathrm{K}^{-1} @ 273 \mathrm{~K}\right)^{[14]}$ 析出量增加 导致的。

根据公式 $Z T=\alpha^{2} \sigma T / \kappa$ 计算 $\mathrm{SnS}_{1-x} \mathrm{Cl}_{x}$ 的无量纲热 电优值 $Z T$, 如图 6(d) 所示。功率因子 $P F$ 的提高和 总热导率 $\kappa_{\mathrm{tot}}$ 的降低, 使 $x=0.04$ 样品在 $823 \mathrm{~K}$ 取得 的最大 $Z T$ 为 0.17 , 比 $x=0(Z T \sim 0.1)$ 样品提高了 $70 \%$ 。

\section{3 结论}

机械合金化(MA)结合放电等离子烧结(SPS)工 艺成功制备了 $\mathrm{n}$ 型 $\mathrm{SnS}_{1-x} \mathrm{Cl}_{x}$, 并得到如下结论:

1)随着 $\mathrm{Cl}^{-}$的加入, $\mathrm{Cl}^{-}$置换 $\mathrm{S}^{2-}$ 提供电子, 使 $\mathrm{SnS}$ 由本征 $\mathrm{p}$ 型转变为 $\mathrm{n}$ 型半导体;

2)泽贝克系数绝对值 $|\alpha|$ 较 $x=0$ 样品增大, 功率因 子 $P F$ 提高 $(x \geqslant 0.03)$; 同时引入点缺陷使热导率 $\kappa_{\mathrm{tot}}$ 降低 $(x \geqslant 0.02)$, 使 $x=0.04$ 样品在 $823 \mathrm{~K}$ 下取得的最大 $Z T$ 为 0.17 , 相比于 $x=0$ 样品 $(Z T \sim 0.1)$ 提高了 $70 \%$;
3)电子浓度在 $10^{15}$ 量级, 远低于高性能热电材料 所要求的最佳载流子浓度水平。如果能进一步提高 掺杂效率，有望实现 $Z T$ 值的显著提升。

\section{参考文献:}

[1] BELL L E. Cooling, heating, generating power, and recovering waste heat with thermoelectric systems. Science, 2008, 321(5895): 1457-1462.

[2] SNYDER G J, TOBERER E S. Complex Thermoelectric Materials. Materials For Sustainable Energy: A Collection of Peer-Reviewed Research and Review Articles from Nature Publishing Group, World Sueutific, 2011: 101-110.

[3] TAN QING, ZHAO LI-DONG, LI JING-FENG, et al. Thermoelectrics with earth abundant elements: low thermal conductivity and high thermopower In doped SnS. Journal of Materials Chemistry A, 2014, 2(41): 17302-17306.

[4] ZHOU BIN-QIANG, LI SHUAI, LI WEN, et al. Thermoelectric properties of $\mathrm{SnS}$ with Na-doping. ACS Applied Materials \& Interfaces, 2017, 9(39): 34033-34041.

[5] WU HONG, LU XU, WANG GUO-YU, et al. Sodium-doped tin sulfide single crystal: a nontoxic earth-abundant material with high thermoelectric performance. Advanced Energy Materials, 2018, 8(20): $1800087-1-8$.

[6] HE WEN-KE, WANG DONG-YANG, DONG JIN-FENG, et al. 
Remarkable electron and phonon band structures lead to a high thermoelectric performance $Z T>1$ in earth-abundant and ecofriendly SnS crystals. Journal of Materials Chemistry A, 2018, 6(21): 10048-10056.

[7] GUO RUI-QIANG, WANG XIN-JIANG, KUANG YOU-DI, et al. First-principles study of anisotropic thermoelectric transport properties of IV-VI semiconductor compounds SnSe and SnS. Physical Review B, 2015, 92(11): 115202-1-13.

[8] WANG XUE, XU JING-TAO, LIU GUO-QIANG, et al. Optimization of thermoelectric properties in n-type $\mathrm{SnSe}$ doped with $\mathrm{BiCl}_{3}$. Applied Physics Letters, 2016, 108(8): 083902-1-5.

[9] CHANG CHENG, TAN QING, PEI YAN-LING, et al. Raising thermoelectric performance of n-type $\mathrm{SnSe}$ via $\mathrm{Br}$ doping and $\mathrm{Pb}$ alloying. RSC Advances, 2016, 6(100): 98216-98220.

[10] ZHANG QIAN, CHERE E K, SUN JING-YING, et al. Studies on thermoelectric properties of n-type polycrystalline $\mathrm{SnSe}_{1-x} \mathrm{~S}_{x}$ by iodine doping. Advanced Energy Materials, 2015, 5(12): 1500360-1-8.

[11] TAN QING, LI JING-FENG. Thermoelectric properties of Sn-S bulk materials prepared by mechanical alloying and spark plasma sintering. Journal of Electronic Materials, 2014, 43(6): 2435-2439.

[12] SITUMORANG M, GOLDSMID H J. Anisotropy of the Seebeck coefficient in bismuth telluride. Physica Status Solidi (b), 1986, 134(1): K83-K88.

[13] GE ZHEN-HUA, ZHANG BO-PING, LI JING-FENG. Microstructure composite-like $\mathrm{Bi}_{2} \mathrm{~S}_{3}$ polycrystals with enhanced thermoelectric properties. Journal of Materials Chemistry, 2012, 22(34): 17589-17594.

[14] NEUBRONNER M, BODMER T, HÜBNER C, et al. D6 Properties of Solids and Solid materials. VDI Heat Atlas. Springer, Berlin, Heidelberg, 2010: 551-614. 